\title{
Etudes expérimentales des profils cross-shore de plages sableuses
}

\author{
Florent GRASSO ${ }^{1}$, Eric BARTHELEMY ${ }^{1}$, Raphaël CERTAIN ${ }^{2}$, \\ Hervé MICHALLET ${ }^{1}$ \\ ${ }^{1}$ CNRS-UJF-INPG, LEGI, BP 53, 38041 Grenoble Cedex 9, France \\ florent.grasso@.hmg.inpg.fr \\ eric.barthelemy@hmg.inpg.fr \\ herve.michallet@.hmg.inpg.fr \\ 2 IMAGE EA4218, Université de Perpignan, 52 av. Alduy, \\ 66000 Perpignan Cedex, France \\ certain@univ-perp.fr
}

\section{Résumé :}

Les principaux objectifs de cette étude sont d'une part de tester en canal le concept d'équilibre morphologique pour un climat de houle donné. Nos expériences se sont déroulées dans le canal à houle à fond sédimentaire du LEGI. Nous avons pu mettre en évidence huit climats de houle menant à un équilibre morphologique. Des profils d'équilibre ont pu être classés en fonction du nombre de Dean. D'autre part, l'évolution des profils de fond reproduit bien les différents scénarios que nous observons en nature, tels que l'érosion ou l'accrétion sédimentaire du haut de plage et les migrations de barres.

\begin{abstract}
:
The main objectives of this study are on one end to test with a physical model the concept of equilibrium beach profile for a wave climate. Our experiments were conducted in the sedimentary LEGI wave-flume. We were able to identify eight wave climates leading to an equilibrium. Equilibrium profiles have been sorted according to their Dean number. On the other end, the evolution of profiles correctly reproduces the different scenarios observed in nature, such as erosion and sediment accretion at the shore and bar migrations.
\end{abstract}

\section{Mots-clés :}

Houle irrégulière - morphodynamique - profils d'équilibre - canal à houle. 


\section{$1 \quad$ Introduction}

La morphodynamique des plages sableuses est au centre du problème de l'érosion des littoraux. La direction des flux sédimentaires cross-shore est un point clé des outils de prédiction et il est fortement corrélé aux caractéristiques non-linéaires des vagues incidentes, comme l'asymétrie et le skewness (Bailard ${ }^{1}, 1981$; da Silva et al. $\left.{ }^{5}, 2006\right)$. Cette complexité est très difficile à reproduire avec des modèles numériques, ainsi la modélisation physique devient une alternative intéressante.

Les outils habituels utilisés en ingénierie pour l'évolution cross-shore considèrent des profils d'équilibre (Dean ${ }^{8}, 1991$; Miller \& Dean $\left.{ }^{21}, 2004\right)$ comme profils cibles. Ces profils se justifient par la notion d'énergie de dissipation volumique des vagues uniforme en zone de $\operatorname{surf}\left(\right.$ Dean $^{7}, 1977$; Wang et al. ${ }^{23}$, 2003). Pour les profils de plage à l'équilibre, le flux sédimentaire moyen crossshore est nul. Partant de cet état de fait Bowen ${ }^{3}$ (1980) et Dronker ${ }^{11}$ (2005), utilisant une estimation simplifiée du flux sédimentaire, montrent que ces profils sont aussi compatibles avec un équilibre entre une force destructive comme la gravité et une force constructive comme le transport sédimentaire par charriage dû aux vagues. Ainsi, la notion de profil de plage à l'équilibre semble raisonnable. Un de nos objectifs est de tester ce concept d'équilibre morphologique avec un modèle physique, sachant que la plupart des études en laboratoire se sont focalisées sur l'érosion ou la formation de barre en zone littorale (Dette \& Uliczka $^{9}, 1987$; Dette et al. ${ }^{10}, 2002$; Dally $\left.{ }^{6}, 1987\right)$.

$\mathrm{Du}$ fait du perpétuel changement de climat de houle en nature, les profils d'équilibre peuvent être retrouvés à partir de profils moyens annuels. Différents profils d'équilibre peuvent correspondre à chaque saison, reliés à un climat de houle défini (Larson \& $\operatorname{Kraus}^{19}$, 1994). Les expériences en canal peuvent surmonter l'inconvénient de définir un profil de plage à l'équilibre comme une moyenne temporelle. Pour des temps d'expérience suffisant, des profils d'équilibre pourront être atteints (Grasso et al. ${ }^{14}, 2007$; Michallet et al. ${ }^{20}, 2007$; Wang \& Kraus $^{22}, 2005$; Kamalinezhad ${ }^{17}, 2004$; Kamalinezhad ${ }^{18}$, 2004).

Les profils de plages sont plus compliqués qu'un simple profil d'équilibre de Dean. Ils tendent à avoir une convexité proche du trait de côte et une partie concave plus au large. Ces profils de type terrasse sont observés dans les environnements dominés par la houle (Wright \& Short ${ }^{24}$, 1984). Les barres au large sont également fréquemment observées sur les profils de plage. 


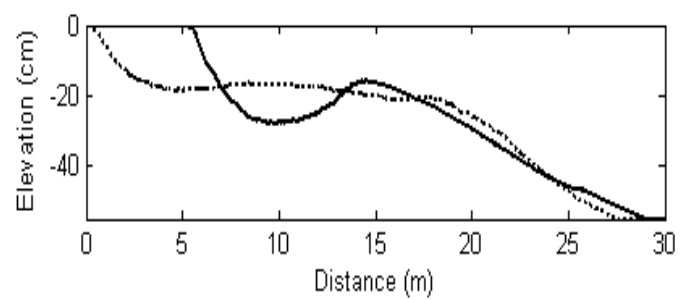

(a) Canal du LEGI

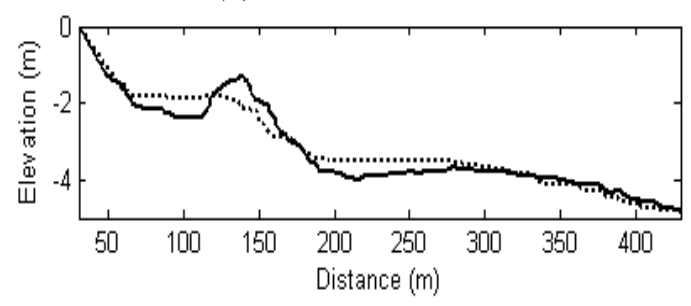

(c) Plage de Sète

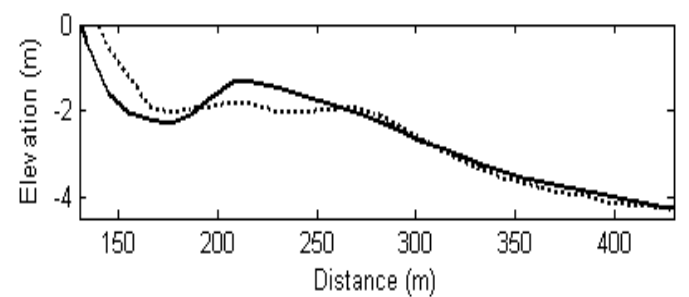

(b) Plage de Duck

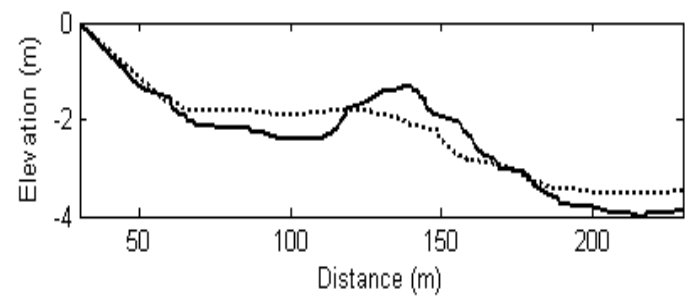

(d) Plage de Sète

Figure 1. Profils de fond : a) canal LEGI b) Duck, 20 oct 1994 Y1250 (-) et 7 septembre 1994 Y1113 (...) c) et d) Sète, 25 nov 2000 (一) et 1 nov 2000 (...).

Nous avons examiné deux sites : la plage du Lido à Sète (Certain \& Barusseau ${ }^{4}$, 2005) et celle de Duck en Caroline du Nord (campagne Duck94). Ces plages sont caractérisées par une houle principalement frontale et par de faibles variations long-shore du profil de fond. Elles se rapprochent ainsi des profils de plage crossshore expérimentaux barrés ou de type terrasse (Figure 1). Nous pouvons définir, à l'échelle de l'année, une profondeur de fermeture de la plage de Sète vers $-5 \mathrm{~m}$ qui se situe à environ $400 \mathrm{~m}$ du trait de côte (Figure 1.c), et vers $-4.5 \mathrm{~m}$ à $300 \mathrm{~m}$ du trait de côte pour la plage de Duck (Figure 1.b). En appliquant une réduction d'échelle géométrique de l'ordre de $1 / 10$, nous remarquons une forte similitude entre les profils de fond des plages naturelles et ceux obtenus en canal (Figure 1.a). Cela est particulièrement visible en terme de positionnement de la barre par rapport au trait de côte et des profondeurs d'eau au niveau de sa crête et son creux.

Notre étude vise à mieux comprendre les liens entre les caractéristiques de la houle et l'évolution long terme de la morphologie des plages. Très peu d'expériences ont été menées sur ces questions car elles requièrent une durée très longue (plusieurs dizaines d'heures en laboratoire, Kamalinezhad ${ }^{17}$, 2004). Selon Wright \& Short $^{24}$ (1984) le nombre de Dean permet de classer les profils de plage cross-shore, nous pouvons le calculer de la manière suivante :

$\Omega=\frac{H_{s}}{T_{p} w_{s}}$

où $H_{s}$ est la hauteur significative des vagues au batteur, $T_{p}$ la période pic du 
spectre des vagues et $w_{s}$ la vitesse de chute du sédiment. Ce nombre peut être interprété comme la mobilisation de la particule par les vagues pendant sa chute. Pour un même nombre de Dean, deux profils de fond seraient similaires. Pour nos expériences, $\Omega<5$, ce qui dans la classification de Wright \& $\operatorname{Short}^{24}$ (1984) correspond à des plages dites réflectives et intermédiaires, caractérisées par des barres long-shore ou cycliques, des terrasses à double convexité (Black et al. ${ }^{2}$, 2002) et des profils raides uniformes.

\section{Dispositif expérimental}

Les expériences se sont déroulées dans un canal de $36 \mathrm{~m}$ de long et $55 \mathrm{~cm}$ de large équipé d'un générateur de vague de type «piston ». La profondeur d'eau au batteur est de $55.3 \mathrm{~cm}$. Le fond du canal est constitué de sédiment mobile de faible densité $\left(\varrho=1.19 \mathrm{~g} / \mathrm{cm}^{3}\right)$ ayant un diamètre moyen $\mathrm{d}_{50}=0.6 \mathrm{~mm}$. Le lit sédimentaire recouvre un faux fond rigide pour certains essais. Les nombres de Froude, Shields et Rouse sont du même ordre de grandeur que ceux des plages naturelles. Les échelles de temps et de longueur sont environ $1 / 3$ et $1 / 10$. Des houles irrégulières sont générées (spectre d'énergie de type JONSWAP - facteur de pic $=3.3$ ). Nous vérifions que ces vagues respectent la distribution de Rayleigh à 2 $\mathrm{m}$ du batteur. Douze sondes à vagues (acquisition à $50 \mathrm{~Hz}$ ), montées sur des chariots, enregistrent l'élévation instantanée de la surface libre et permettent de mesurer la hauteur quadratique $H_{r m s}\left(H_{r m s} \sim 0.7 H_{s}\right)$ et la période pic des vagues $\left(T_{p}\right)$ (Tableau 1). Les profils de fond sont relevés entre chaque série de vagues grâce à un profileur acoustique monté sur un chariot motorisé.

Tableau 1. Climats de houle générés dans le canal, utilisés pour cette étude.

\begin{tabular}{|c|c|c|c|c|c|c|}
\hline Série & $\begin{array}{c}\boldsymbol{H} \boldsymbol{s} \\
(\mathbf{m m})\end{array}$ & $\begin{array}{c}\boldsymbol{T p} \\
\mathbf{( s )}\end{array}$ & $\boldsymbol{\Omega}$ & $\begin{array}{c}\text { Durée } \\
\text { (h) }\end{array}$ & $\begin{array}{c}\text { Temps pour atteindre } \\
\text { l'équilibre (h) }\end{array}$ & Type \\
\hline $\mathrm{A}$ & 30 & 2 & 0.8 & 9 & 6 & estival \\
\hline $\mathrm{B}$ & 60 & 1.5 & 2 & 82 & 70 & \\
\hline $\mathrm{C} 1$ & 107 & 2 & 2.7 & 41 & 25 & tombant de tempête \\
\hline $\mathrm{C} 2$ & 107 & 2 & 2.7 & 52 & 35 & tombant de tempête \\
\hline D & 160 & 3 & 2.7 & 115 & 45 & tempête \\
\hline E & 107 & 1.4 & 3.9 & 7 & 4 & \\
\hline F & 125 & 1.6 & 4 & 33 & 30 & \\
\hline G & 160 & 2 & 4.1 & 30 & 30 & \\
\hline
\end{tabular}




\subsection{Profils de fond à l'équilibre}

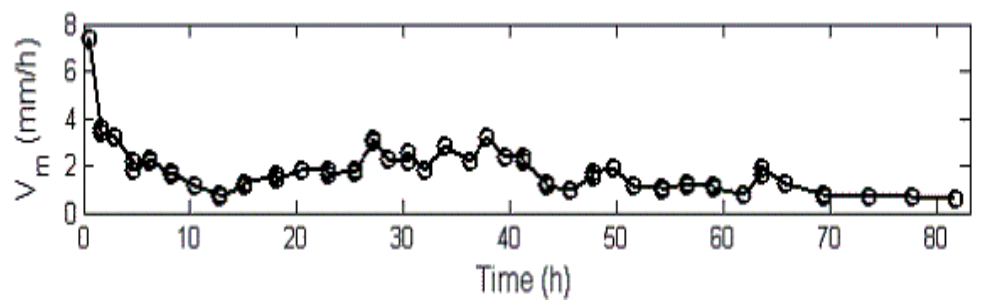

Figure 2. Vitesse moyenne d'évolution des profils de fond calculée pour la série de vagues $B$.

L'état d'équilibre du fond est déterminé par le calcul de la vitesse moyenne:

$$
V_{m}=\frac{1}{l_{p}} \int^{l_{p}}\left|\frac{d h_{s}}{d t}\right| d x
$$

où $l_{p}$ est la longueur du profil de fond étudié, $h_{s}$ la hauteur de sédiment le long du profil et $t$ la durée de génération des vagues. Nous estimons avoir atteint l'équilibre lorsque $V_{m} \sim 1 \mathrm{~mm} / \mathrm{h}$ (environ 70 heures pour la série B, cf. Figure 2). Nous obtenons ainsi huit conditions d'équilibre morphologique (Figure 3).

Le temps nécessaire à l'obtention des états d'équilibre peut être plus ou moins long en fonction de l'éloignement du profil de fond initial au profil de fond final (par exemple pour A et E l'équilibre est très vite atteint: 6 et $4 \mathrm{~h}$ ). Notons que la présence du fond fixe ne semble pas limiter l'érosion et influencer la dynamique sédimentaire (profils similaires Figure 3.f, g et $\mathrm{h}$ ). Pour des profils de fond initiaux différents, les profils finaux à l'équilibre sont fortement similaires pour des conditions identiques $(\mathrm{C} 1$ et $\mathrm{C} 2)$. Nous retrouvons le plateau à la même profondeur et les pentes de bas et de haut de plages sont identiques, avec toutefois une différence de positionnement du trait de côte (Figure 3.c, d). L'évolution de profils de fond différents soumis au même climat de houle ne sont pas exactement reproductibles mais similaires en termes de forme. L'action des vagues est limitée à une certaine profondeur, définissant ainsi une plage d'influence. La similarité de forme des profils à l'équilibre pour un climat de houle donné serait donc correcte sur la partie du profil délimitée par la profondeur d'action de cette houle. Cela est clairement visible sur la Figure 3.a: seule la partie haute du profil de fond est remodelée par la série $\mathrm{A}$ (pour une profondeur d'eau inférieure à environ $20 \mathrm{~cm}$ ). 


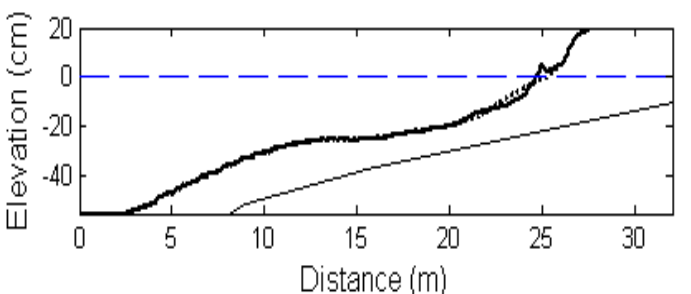

(a) Série A, $\Omega=0.8$

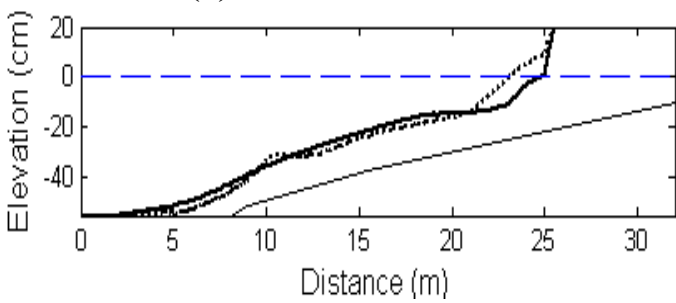

(c) Série $\mathrm{C} 1, \Omega=2.7$

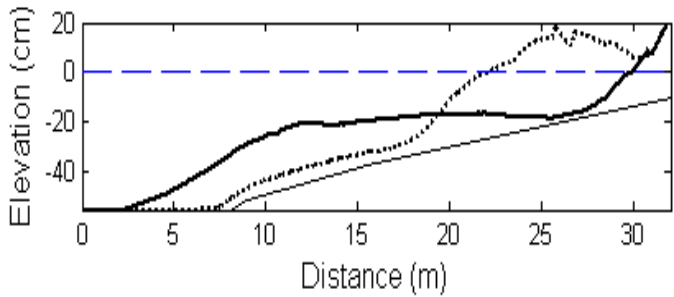

(e) Série $\mathrm{D}, \Omega=2.7$

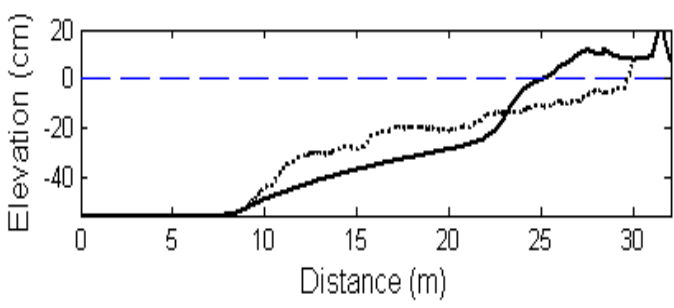

(g) Série F, $\Omega=4$

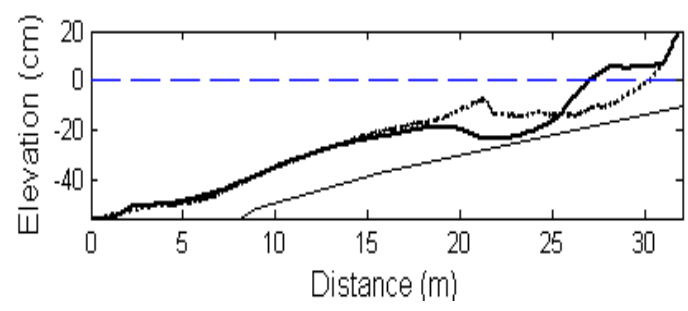

(b) Série $\mathrm{B}, \Omega=2$

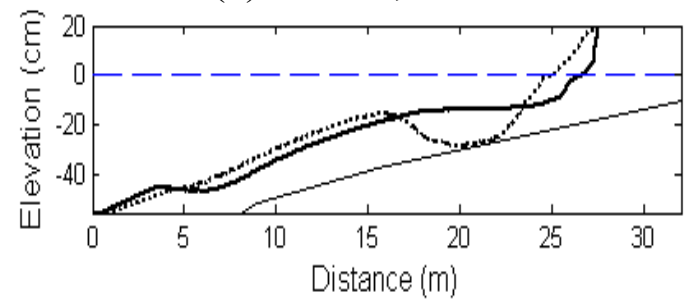

(d) Série $C 2, \Omega=2.7$

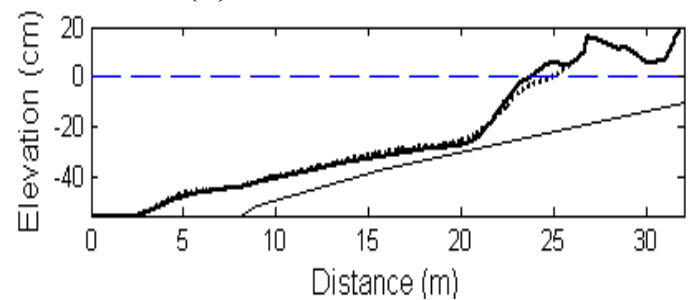

(f) Série E, $\Omega=3.9$

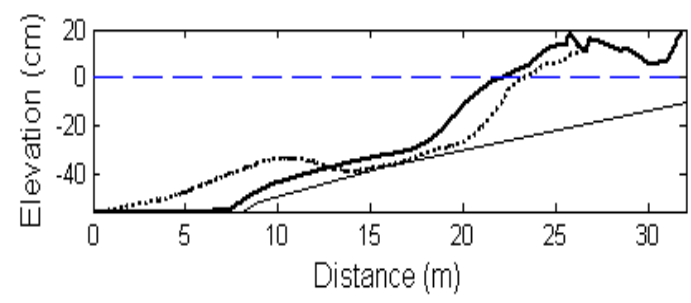

(h) Série G, $\Omega=4.1$

Figure 3. Profils de fond initiaux (...) et finaux à l'équilibre (-) pour huit séries de houles.

\subsection{Classification des profils d'équilibre en fonction du nombre de Dean}

Wright \& Short ${ }^{24}$ (1984) ont présenté une classification des profils de plage en fonction de $\Omega(1)$ : les plages dissipatives caractérisées par de faibles pentes et un déferlement glissant $(\Omega>6)$, les plages intermédiaires de pentes modérées et un déferlement plongeant $(1<\Omega<6)$, les plages réflectives à fortes pentes et déferlement principalement frontal (ou gonflant) pour $\Omega<1$. Nos expériences permettent de simuler des climats de houle dont le nombre de Dean est compris entre 0.8 et 4.1 . Pour les séries A et B (Figure 3.a et b), le bas de plage est considéré comme immobile car l'influence des vagues est limitée aux environs de 20 et $30 \mathrm{~cm}$ de profondeur, le profil global est donc peu représentatif du nombre 
de Dean. Les profils de fond à l'équilibre des Figure 3.c, $d$ et e correspondent aux séries $\mathrm{C} 1, \mathrm{C} 2$ et $\mathrm{D}$ pour lesquelles $\Omega=2.7$ : des profils de type terrasse dont la profondeur varie avec l'amplitude de la houle. La pente moyenne des profils (calculée du trait de côte au pied de plage) est de l'ordre de $1 / 45$. Pour les profils d'équilibre des Figure 3.f, g et $\mathrm{h}(\mathrm{E}: \Omega=3.9$, F: $\Omega=4$ et $\mathrm{G}: \Omega=4.1$, nous remarquons des formes très similaires caractérisées par une partie très raide en haut de plage qui s'adoucit ensuite en profondeur. Cela se traduit par des pentes moyennes plus raides avoisinant $1 / 30$. Les profils de plage raides associés à un $\Omega<1$ s'expliquent par un temps de chute du sédiment très court devant la période pic des vagues, privilégiant ainsi le transport sédimentaire par charriage. Le profil est donc influencé par l'association de la granulométrie (à travers la vitesse de chute) et de l'hydrodynamique. Les processus sont plus complexes lorsque le temps de chute du sédiment devient plus important $(\Omega>1)$, une particule peut être mobilisée par plusieurs vagues et la présence d'un courant de retour plus ou moins établi pourra influencer la distribution sédimentaire. Nous retrouvons dans ces expériences que les plages intermédiaires peuvent êtres vues comme la composition d'une partie réflective et d'une autre dissipative.

\subsection{Profils de fond transitoires}

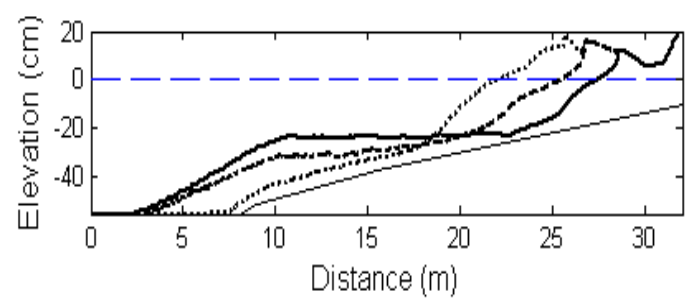

(a) $D$ à $t=0$ (...), $t=11.2 \mathrm{~h}(--)$ et $t=20.2 \mathrm{~h}($ (-)

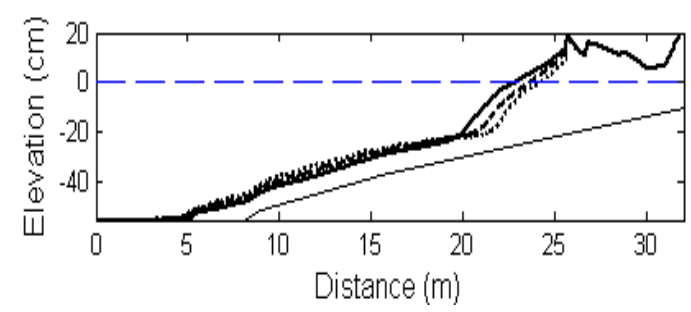

(b) $G$ à $t=0$ (..), $t=3.3 \mathrm{~h}(--)$ et $t=7.4 \mathrm{~h}(-)$

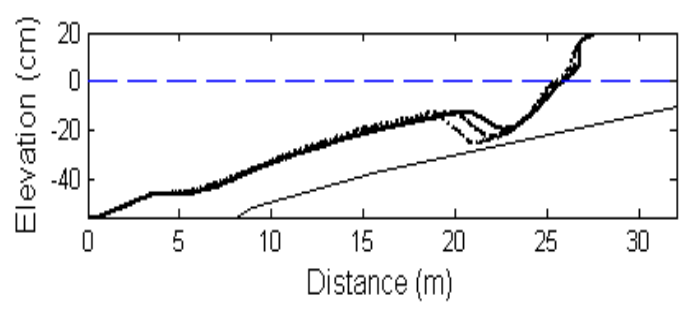

(c) $C 2$ à $t=0$ (...), $t=5.4 \mathrm{~h}(--)$ et $t=9.6 \mathrm{~h}(-)$

Figure 4. Profils de fond transitoires caractéristiques : érosion du haut de plage (a), accrétion du haut de plage (b) et migration de barre vers la côte (c).

Un profil de fond est défini comme «transitoire » quand sa morphologie évolue en fonction du temps pour un climat de houle généré, cela se visualise par une 
vitesse moyenne d'évolution importante. Prenons l'exemple de la Figure 2, suite à un important pic d'intensité $(7.4 \mathrm{~mm} / \mathrm{h})$ la vitesse moyenne suit une forte décroissance pendant les 13 premières heures d'expérience chutant à $0.7 \mathrm{~mm} / \mathrm{h}$, puis cette vitesse augmente à nouveau vers $3 \mathrm{~mm} / \mathrm{h}$ par pics successifs. Ces variations de vitesse d'évolution sont liées à la formation de barres et leur migration vers la côte.

Nous présentons sur la Figure 4 trois scénarios d'évolutions caractéristiques menant chacun à un état d'équilibre. La Figure 4.a représente une phase d'érosion $\mathrm{du}$ haut de plage avec la formation d'un plateau horizontal, qui correspond au passage d'un climat de houle de fort nombre de Dean (série $G, \Omega=4.1$ ) à un plus faible (série $D, \Omega=2.7$ ). Cela se traduit par un transport sédimentaire du haut de plage vers le bas, qui vient adoucir la pente moyenne. Nous retrouvons ce type de scénario en nature pour les cas de tempêtes où le profil de haut de plage s'érode et bascule pour former une terrasse plus en profondeur (Günaydin \& Kabdasli ${ }^{13}$, 2003).

Le scénario inverse est représenté sur la Figure 4.b avec une accrétion du haut de plage au détriment du bas. Le nombre de Dean fort $(\Omega=4.1)$ de la série de houle générée $(\mathrm{G})$ tend à raidir le profil de fond, le sédiment est mobilisé dès le pied de plage pour aller engraisser le haut et faire avancer le trait de côte vers le large. Cela est typique des observations faites sur la reconstruction d'un profil de plage après une forte érosion (Certain \& Barusseau ${ }^{4}, 2005$ ).

Enfin, la Figure 4.c met en avant le cas d'une migration de barre vers la côte, sous l'action des vagues $\mathrm{C} 2$ la barre se déplace à la manière de la propagation d'une dune avec une asymétrie de forme entre la pente amont (plus raide) et aval. La barre vient alors se coller en haut de plage, suite à un temps de génération de vagues suffisamment long. La dynamique des barres sédimentaires a été largement observée en nature (Gallagher et al. ${ }^{12}$, 1998 ; Hoefel \& Elgar ${ }^{15}$, 2003 ; Hsu et al. ${ }^{16}, 2006$ ), mais leur discrétisation temporelle reste très limitée. Ainsi, les cas de migration de barre, n'ayant à notre connaissance jamais été simulés auparavant en laboratoire, apportent de nombreuses informations sur la dynamique des plages naturelles.

\section{$4 \quad$ Conclusions}

Un des principaux objectifs de cette étude était de tester en canal le concept d'équilibre morphologique pour un climat de houle donné. Grâce à l'utilisation d'un sédiment de faible densité permettant de respecter les similitudes hydrosédimentaires, nous reproduisons en laboratoire (échelle de réduction géométrique d'environ 1/10) des profils de plages observés en nature (lido de Sète et plage de Duck). 
Un équilibre morphologique a été atteint pour huit climats de houle. Nous avons également établi une classification des profils de fond à l'équilibre en fonction du nombre de Dean $(\Omega)$ : il apparaît clairement une similarité de forme des profils de type « terrasse » pour $\Omega=2.7$ et des profils raides et plus uniformes pour $\Omega \sim 4$.

Les profils de fond en régime transitoire reproduisent des scénarios observés en nature : des phases d'érosion ou d'accrétion du haut de plage, mais aussi des cas de formations de barres et leurs migrations vers la côte. Le couplage des différents processus hydrodynamiques (undertow, asymétrie des vagues) et sédimentaires (charriage, suspension) étant particulièrement complexe, ces expériences présentent ainsi un intérêt pour la calibration des modèles numériques de morphodynamique.

\section{$5 \quad \underline{\text { Remerciements }}$}

Cette étude a bénéficié du support technique de Jean-Marc Barnoud, de financements LITEAU II et INTERREGIV-Beachmed-e et de données recueillies par l'US Army Corps of Engineers lors de la campagne Duck94 (http://www.frf.usace.army.mil/duck94/DUCK94.stm).

\section{$6 \quad$ Références bibliographiques}

[1] J.A. Bailard. An energetics total load sediment transport model for a plane sloping beach. J. Geophys. Res., 86(C11):10938-10954, 1981.

[2] K.P. Black, R.M. Gorman, K.R. Bryan. Bars formed by horizontal diffusion of suspended sediment. Coastal Engineering, 47(1):53-75, 2002.

[3] A.J. Bowen. Simple models of nearshore sedimentation; beach profiles and longshore bars. In McCann S.B., editor, The Coastline of Canada, volume Paper 80-10:1-11. Geological Survey of Canada, 1980.

[4] R. Certain, J.-P. Barusseau. Conceptual modelling of sand bars morphodynamics for a microtidal beach (Sète, France). Bull. Soc. géol. Fr., t.176, no 4, pp. 343-354, 2005.

[5] P.A. da Silva, A. Temperville, F. Seabra Santos. Sand transport under combined current and wave conditions: a semi-unsteady, practical model. Coastal Engineering, 53:897-913, 2006.

[6] W.R. Dally. Longshore bar formation. In Coastal Sediments'87, ASCE, 71-86, New Orleans, Louisianna, USA, 1987.

[7] R.G. Dean. Equilibrium beach profiles: U.S. Atlantic and Gulf coasts. Ocean engineering report no. 12, Department of Civil Engineering, University of Delaware, 1977.

[8] R.G. Dean. Equilibrium beach profiles: characteristics and applications. J. Coastal Res., 7(1):53-84, 1991.

[9] H. Dette, K. Uliczka. Prototype investigation on time-dependent dune recession and beach erosion. In Coastal Sediments'87, ASCE, 1430-1444, New Orleans, Louisianna, USA, 1987. 
[10] R.G. Dette, M. Larson, J. Murphy, J. Newe, K. Peter, A. Reniers, H. Steetzel. Application of prototype flume tests for beach nourishment assessment. Coastal Engineering, 47:137-177, 2002.

[11] J. Dronkers. Dynamics of Coastal Systems, volume 25 of Advanced Series on Ocean Engineering. World Scientific, pp.387-414, 2005.

[12] E.L. Gallagher, S. Elgar, R.T. Guza. Observation of sand bar evolution on a natural beach. J. Geophys. Res., 193(C2):3203-3215, 1998.

[13] K. Günaydin, M.S. Kabdasli. Characteristics of coastal erosion geometry under regular and irregular waves. Ocean Engineering, 30:1579-1593, 2003.

[14] F. Grasso, H. Michallet, E. Barthélemy. Infragravity waves in modile-bed laboratory experiments. In Coastal Sediment 07, ASCE, New Orleans, Louisianna, USA, 2007.

[15] F. Hoefel, S. Elgar. Wave-induced sediment transport and sandbar migration. Science, 299:1885, 2003.

[16] T.-J. Hsu, S. Elgar, R.T. Guza. Wave-induced sediment transport and onshore sandbar migration. Coastal Engineering, 53:817-824, 2006.

[17] M. Kamalinezhad. Plages en équilibre morphologique et hydrodynamique associée. PhD Thesis, Institut National Polytechnique de Grenoble, France, 2004.

[18] M. Kamalinezhad. Equilibre morphologique de barres de déferlement: expériences. VIII ${ }^{\mathrm{e}}$ Journées Nationales Génie Civil - Génie Côtier, Compiègne, France, 2004.

[19] M. Larson, N.C. Kraus. Temporal and spatial scales of beach profile change, Duck, North Carolina. Marine Geology, 117:75-94, 1994.

[20] H. Michallet, F. Grasso, E. Barth_elemy. Long waves and beach evolution profiles. J. Coastal Res., SI50, 221-225, 2007.

[21] J.K. Miller, R.G. Dean. A simple new shoreline change model. Coastal Engineering, 51:531-556, 2004.

[22] P. Wang, N.C. Kraus. Beach profile equilibrium and patterns of wave decay and energy dissipation across the surf zone elucidated in a largescale laboratory experiment. Journal Coastal Research, 21(3):522-534, 2005.

[23] T. Wang, B.A. Ebersole, E.R. Smith. Beach-profile evolution under spilling and plunging breakers. J. Waterway, Port, Coastal and Ocean Eng., 129(1):41-46, 2003.

[24] L.D. Wright and A.D. Short. Morphodynamic variability of surf zones and beaches: a synthesis. Marine Geology, 56:93-118, 1984. 